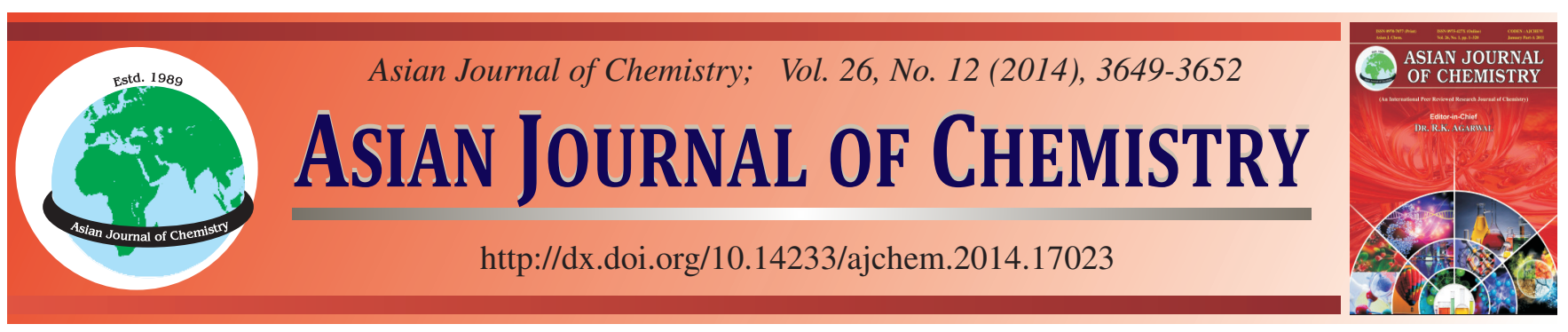

\title{
Physico-chemical Characterization of Microemulsions Containing Plant Extract Using Non-ionic Surfactant
}

\author{
RASHidA PARVEEN ${ }^{1, *}$ and NAVEed AKhtar ${ }^{2}$
}

${ }^{1}$ School of Pharmacy, The University of Faisalabad, Faisalabad 38000, Pakistan

${ }^{2}$ Department of Pharmacy, Faculty of Pharmacy and Alternative Medicine, The Islamia University of Bahawalpur, Bahawalpur 63100, Pakistan

*Corresponding author: E-mail: rashida.pharmacist@gmail.com

Microemulsions of both blank and with plant extract were formulated by using propylene glycol and Tween 80 as non-ionic surfactant with isopropyl myristate oil and water. The chemical compositions of Tween 80 surfactant strongly affect the stability study of formulation. Surfactant-cosurfactant mixture in ratio of 2:1 $\left(\mathrm{S}_{\text {mix }}\right)$ along with isopropyl myristate (oil) was selected and phase diagrams were constructed. The $n$-hexane extract of Red Carica papaya pulp was prepared and analyzed lycopene by UV-visible spectrophotometric method at 470 $\mathrm{nm}$. The percentage of lycopene was measured by calibration curve. The physico-chemical stability of microemolsions blank and plant extract were evaluated regarding their particle size, zeta potential, conductivity, stability, viscosity, scanning electron microscopy, refractory index (RI) and $\mathrm{pH}$. This study established that a stable microemulsion containing Carica papaya fruit extract was dependent upon the contents of S/C ratio, oil and water in formulations. Physicochemically and physically extract containing microemulsion with $2: 1$ was found to be stable therefore it can be used for topical application to deliver natural antioxidant.

Keywords: Microemulsion, Tween 80, Physicochemical stability, Extract.

\section{INTRODUCTION}

The purpose of the present study is to formulate plant or Carica papya extract in microemulsion carrier systems intended for topically application of incorporated antioxidant actives, flavonoids and polyphenols. Stability studied of extract containing microemusion. Microemulsions are macroscopically isotropic mixtures of at least a hydrophilic, a hydrophobic and an amphiphilic component. Their thermodynamic stability and their nanostructure are two important characteristics that distinguish them from ordinary emulsions which are thermodynamically unstable ${ }^{1}$. Non ionic surfactant polysorbate 80 (Tween 80) has been widely used in the preparations of drugs, cosmetics, skin care products and food as a solubilizer, emulsifier and stabilizer. Polysorbate 80 has been shown to have a typical structure (Fig. 1) that contains approximately 20 groups of polyoxyethylene (POE) per molecule ${ }^{2}$. The surfactant's physicochemical behaviour of polysorbate 80 , such as the hydrophilic lipophilic balance (HLB) or the critical micelle concentration (CMC) value, is dependent upon its chemical composition, governed by the structure of the sorbitol derivative core (i.e., sorbitan and isosorbide), the degree of esterification, the number of polyoxyethylene groups and their distribution within the molecule ${ }^{2}$. The concept of HLB (Hydrophilic-Lipophilic Balance) was introduced by Griffin in 1949. As the name suggests,
HLB is an empirical balance based on the relative percentage of the hydrophilic to the lipophilic moieties in the surfactant. Later, Griffin in year 1954 defined an empirical equation that can be used to determine the HLB based on chemical composition. Davies ${ }^{1}$ has offered a more general empirical equation by assigning a number to the different hydrophilic and lipophilic chemical groups in a surfactant. The HLB number was calculated by the expression,

$$
\mathrm{HLB}=[(\mathrm{nH} \times \mathrm{H})-(\mathrm{nL} \times \mathrm{L})]+7
$$

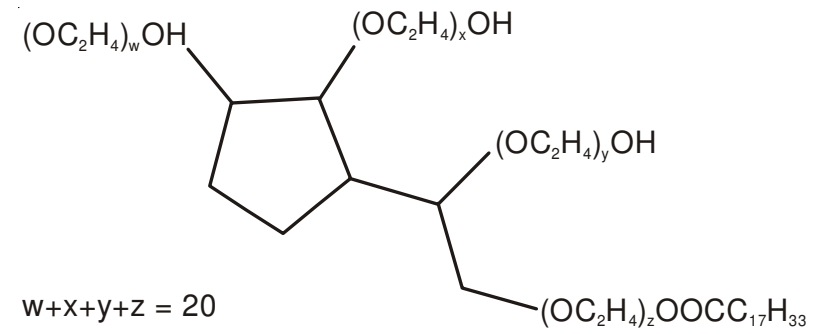

Fig. 1. Typical structure of Polysorbate 80

where $\mathrm{H}$ and $\mathrm{L}$ is the numbers assigned for the hydrophilic and lipophilic groups respectively and $\mathrm{nH}$ and $\mathrm{nL}$ are the respective numbers of these groups per surfactant molecule. in vitro release rate of diclofenac sodium from microemulsions containing soybean oil, nonionic surfactants (Brij 58 and Span 
80) and different alcohols (ethanol, isopropanol and propanol) as cosurfactant. Phase diagrams were constructed to detect the optimum surfactant:cosurfactant $(\mathrm{S}: \mathrm{CoS})$ weight ratios and microemulsion areas. Three microemulsion formulations were selected and their physicochemical properties were studied for the $\mathrm{pH}$, conductivity and viscosity ${ }^{4}$. Red-fleshed papaya fruit contain high levels of lycopene, whereas yellow-fleshed fruit do not. The colour of papaya fruit flesh is determined largely by the presence of carotenoid pigments. Red-fleshed papaya fruit contain lycopene, whilst this pigment is absent from yellow-fleshed fruit ${ }^{5}$. Red fleshed papaya contains lycopene which is potent antioxidant. Cosmetically use of the papaya juice is to help reduce and attenuate freckles or brown spots due to exposure to sunlight, smoothing the skin and creating a healthier looking skin ${ }^{6}$. Topical application of lycopene is a convenient way to restore antioxidants depleted from the skin by UV radiation and achieve protection against premature aging and cancer ${ }^{7}$.

\section{EXPERIMENTAL}

Surfactant or Tween 80 (Fisher Scientific, Germany), Cosurfactant is Propylene Glycol (Merck, Germany) Isopropyl Myristate (Fisher Scientific, Germany), Standard lycopene (Sigma), $n$-hexane extract of Carica papaya, Vortex (Scientific industries, USA)

Preparation of stock solution and analysis at UV-spectrophotometer: The stock standard solution of lycopene was taken and prepared required concentration range of 0.156 to $10 \mu \mathrm{g} / \mathrm{mL}^{8}$. The absorbance of each concentration was observed at UV at $470 \mathrm{~nm}$ and plotted against concentration of lycopene to give the calibration curve (Fig. 2). The calibration curve obtained for the determination of lycopene was $\mathrm{y}=444.99 \mathrm{x}-36.846, \mathrm{R}^{2}=0.997$ for the quantitation of Lycopene in Carica papaya fruit extract, the amount of lycopene was calculated based on absorbance of sample and using the calibration curve. The percentage of lycopene in Carica papaya fruit extract calculated was found to be $70.07 \%$. The correlation coefficient $\left(\mathrm{R}^{2}\right)$ was used to determine the linearity of the calibration curve.

Estimation of lycopene in extract: $1 \mathrm{~mL}$ of extract was mixed with $10 \mathrm{~mL}$ of $n$-hexane and then further diluted to 10 $\mu \mathrm{g} / \mathrm{mL}$. The sample solution was mixed well by using vortex

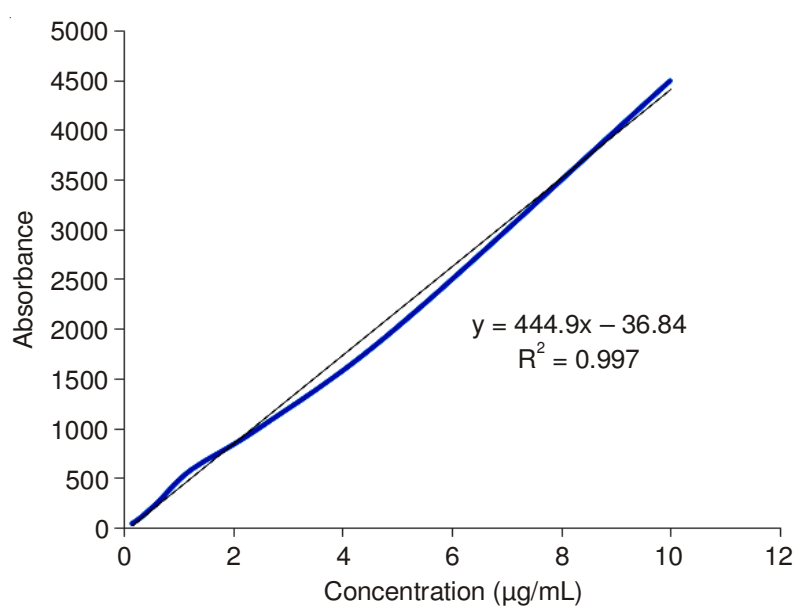

Fig. 2. Calibration curve of lycopene
(Scientific industries, USA) and filtered through $0.45 \mu \mathrm{m}$ cellulose acetatemembrane before analysis. The amount of lycopene in extract was back-calculated from the calibration curve equation.

Pseudoternary phase diagram construction: All microemulsions were formulated with full pseudoternary phase diagram. The surfactant (Polysorbate 80) and co-surfactant (propylene glycol) were used in the preparation of the microemulsion. Mixture of ratios (2:1 and 4:1) of surfactant and cosurfactant mixtures (Fig. 3a and 3b). The microemulsion regions in the pseudoternary phase diagram were determined. The results showed the region of microemulsion on the phase diagram was greater with $2: 1$ ratio. The microemulsions were prepared using the best combination with isopropyl myristate as oil at percentage obtained from preliminary studies by diluting the combination of oil and surfactant mixture with water using a magnetic stirrer for $5 \mathrm{~min}$. The ratio surfactant to cosurfactant was $2: 1$. The concentration of water varied from 5 to $50 \% \mathrm{w} / \mathrm{w}$ in $5 \%$ increment. The mixture turned from turbid through opaque to a translucent yellow mixture and this marked the formation of a microemulsion.

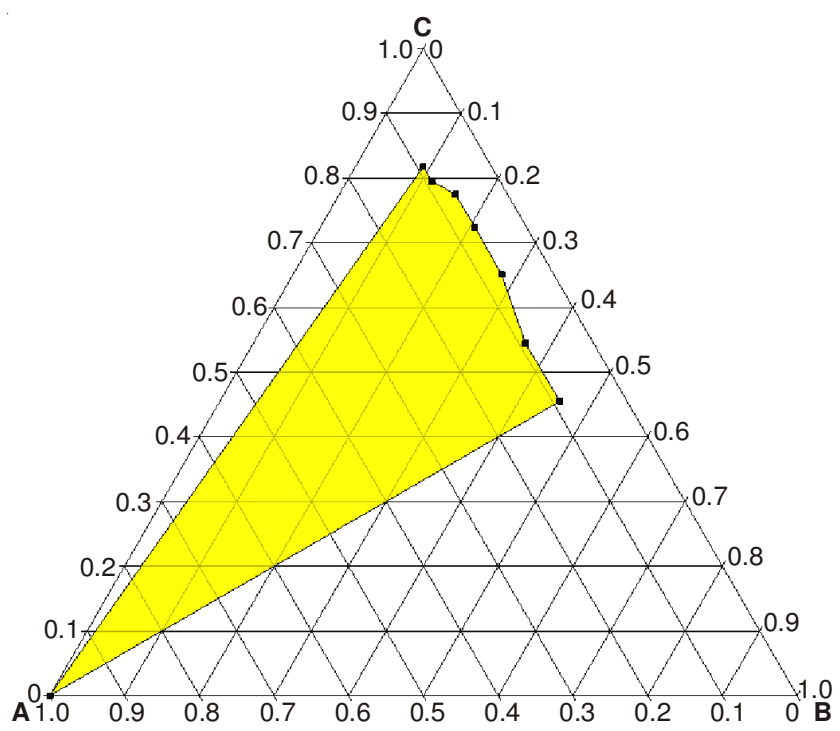

(a)

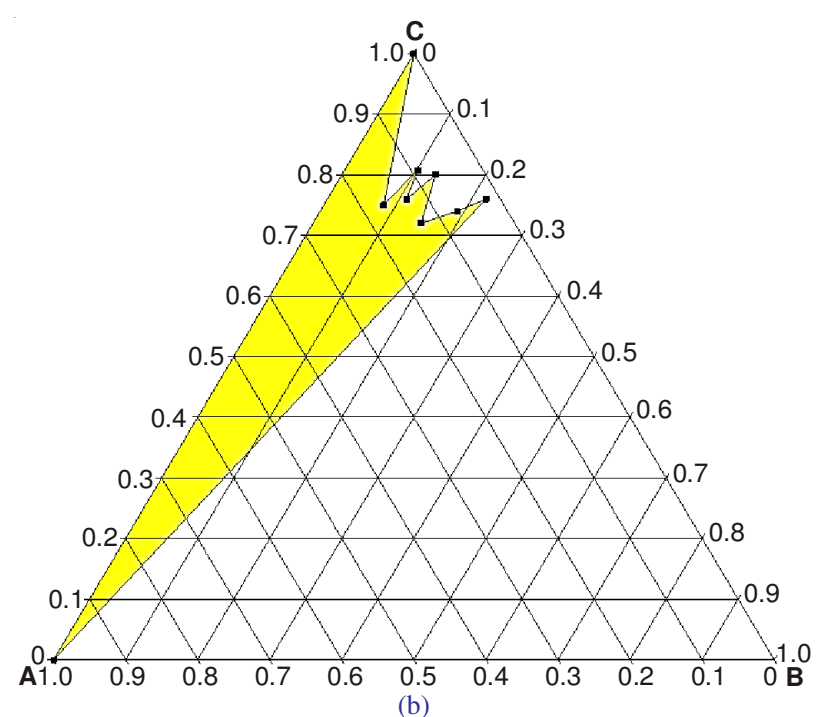

Fig. 3. Pseudoternary phase diagram of surfactant/co-surfactant mixture, oil and water. (a) $S_{\text {mix }} 2: 1$ and (b) $S_{\text {mix }} 4: 1$ 
Zeta potential mearsurement: Zeta potential of samples were measured by Zetasizer (Malvern instrument 1td ZEN3600, UK). Samples were placed in clear disposable zeta cells and results were recorded.

Particle size measurements: The average droplet size of samples was measured at $25^{\circ} \mathrm{C}$ by Scatter Scope 1 Quidix (South Korea)

Viscosity measurements: Viscosity of samples was measured at $25^{\circ} \mathrm{C}$ with a Brookfield viscometer (DV-II+Pro Brookfield., USA) using spindle no. 41. With shear rate 100$200 \mathrm{rpm}$. Each measurement was performed in triplicate.

Refractive index measurements: The refractory indices (RI) of samples were measuredby Abbe refractometer (HEDAO, China).

Scanning electron microscopy (SEM): Scanning electron microscopywas used to characterize microstructure of microemulsions. Scanning electron microscopy of samples was measured by scanning electron microscopy (19800 Hitachi S-3400N VP-SEM, Japan) under variable pressure. The image of the microemulsion containing extract is shown in Fig. 6.

Spreadability: Spreadability of all formulations was measured in terms of diameter. In this method, weighed quantity of formulations i.e. $500 \mathrm{mg}$ was placed on one glass slide and another glass slide of $10 \mathrm{~g}$ weight was placed over the first slide. As a result of compression between two slides, a circle of the formulation was produced and its diameter was measured ${ }^{9}$.

Conductivity measurements: Electrical conductivity of MEs was measured with a conductivity meter (Conductometer WTW, Cond 197i, Germany).All measurements were carried out in triplicate.

Determination of $\mathbf{p H}$ : The $\mathrm{pH}$ values for microemulsion were determined at $25^{\circ} \mathrm{C}$ by $\mathrm{pH}$ meter (Inolab, Germany). All measurements were carried out in triplicate.

Physical stability study: The physical stability of microemulsions was studied regarding the temperature stability and centrifugation. Microemulsions were stored at various temperatures $\left(4,25,40\right.$ and $\left.50^{\circ} \mathrm{C}\right)$ and observed for phase separation, visual clarity flocculation or precipitation for 3 months. Also, applied stress test, microemulsions were centrifuged by high speed centrifuge machine (Helttich, Germany $8000 \mathrm{rpm}$ for $0.5 \mathrm{~h}$ at $25^{\circ} \mathrm{C}$ and inspected for any change in their homogeneity.

Statistical methods: All the experiments were performed in triplicates and data were expressed as the mean value \pm SD. Statistical data were analyzed by one-way analysis of variance (ANOVA) and $\mathrm{P}<0.05$ was considered to be significant with $95 \%$ confidence intervals.

\section{RESULTS AND DISCUSSION}

Pseudo-ternary phase diagrams: Pseudo-ternary phase diagrams of the investigated quaternary system water/isopropyl myristate, polysorbate Tween 80/propylene glycol with different surfactant-co-surfactant ratios are shown in Fig. 3a and $3 \mathrm{~b}$. Microemulsions were formed at ambient temperature. The phase diagrams clearly indicated that microemulsion existence region increased with increase in the weight ratio of surfactant/co-surfactant $\left(S_{\text {mix }}=2: 1\right)$. The larger single-phase region has been observed in Tween 80 systems. The observations can be correlated to the hydrophile-lipophile balance (HLB) values of these surfactant systems. Tween 80 with a HLB value of 15 is most hydrophilic in nature ${ }^{10}$.

Physico-chemical characterization: Globule size of blank microemulsion and microemulsion containing extract were determined and there an increase in average globule size after loading the drug was observed which may be due to coalescence of globules while drug loading. The ME containing extract had the lowest average globule size $52.507 \pm 0.2 \mathrm{~nm}$, so it indicates the uniformity of droplet size within the formulation $^{11}$. Analysis of variance showed that correlation between mean particle size, polydespersity idex and independent variables are not significant $(\mathrm{p}>0.05)$. The $\%$ size distribution is shown in Figs. 4 and 5 of blank and extract containing microemulsions. The size of particles of extract containing microemulsion was further confirmed by scanning electron microscope is shown in Fig. 6.

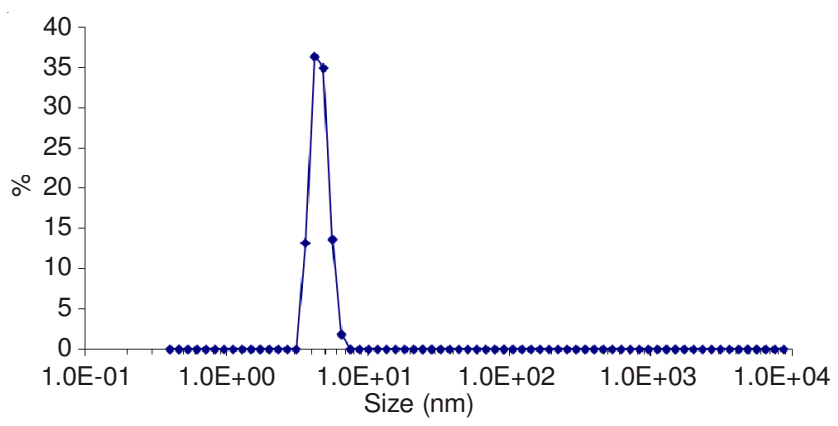

Fig. 4. \% of size distribution of globules in Blank microemulsion

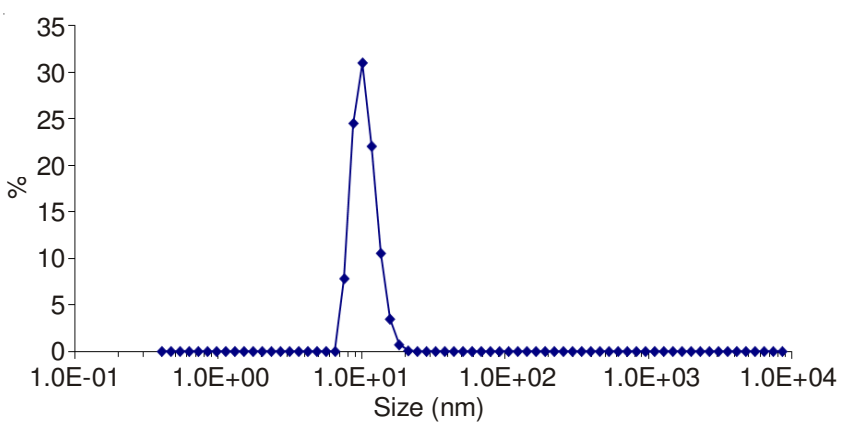

Fig. 5. \% of size distribution of globules in Extract containing microemulsion

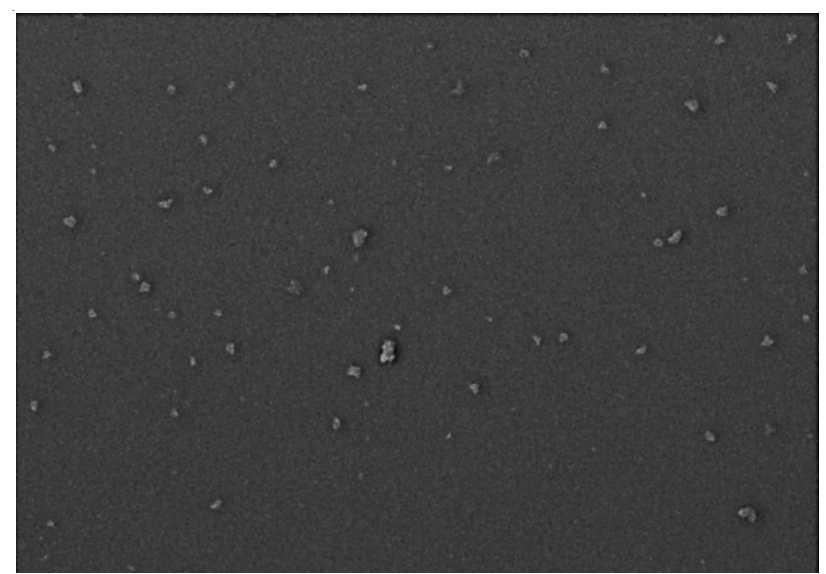

Fig. 6. SEM of microemulsion containing Extract 
TABLE-1

PHYSICO-CHEMICAL CHARACTERISTICS OF BLANK MICROEMULSION AND MICROEMULSION CONTAINING EXTRACT $(\mathrm{n}=3$ )

\begin{tabular}{cccccccccc}
\hline S. No. & Formulations & $\begin{array}{c}\text { Viscosity } \\
(\mathrm{cP})\end{array}$ & $\begin{array}{c}\text { Particle size } \\
(\mu \mathrm{m})\end{array}$ & $\begin{array}{c}\text { Conductivity } \\
(\mu \mathrm{S} / \mathrm{cm})\end{array}$ & $\mathrm{pH}$ & $\mathrm{RI}$ & $\begin{array}{c}\text { Phase } \\
\text { separation }\end{array}$ & $\begin{array}{c}\text { Visual } \\
\text { inspection }\end{array}$ & $\begin{array}{c}\text { Spreadability } \\
(\mathrm{cm})\end{array}$ \\
\hline 1 & $\begin{array}{c}\text { Blank } \\
\text { microemulsion }\end{array}$ & $5.54 \pm 0.12$ & $33.959 \pm 0.4$ & 4.3 & 5.93 & 1.44 & No & Clear & 5.62 \\
2 & $\begin{array}{c}\text { With extract } \\
\text { microemulsion }\end{array}$ & $4.44 \pm 0.05$ & $52.507 \pm 0.2$ & 3.4 & 5.90 & 1.45 & No & Clear & 5.81 \\
\hline
\end{tabular}

The refractive index (RI) of the blank microemulsion and microemulsion containing Carica papaya fruit extract was found 1.41 and 1.45 , respectively which showed that the microemulsion were transparent because most transparent media when viewed ${ }^{12}$ under visible light have refractive indices between 1 and 2 .

There was a strong correlation between the specific structure of the microemulsion systems and their electrical conductive behaviour. The blank microemulsion and microemulsion containing extract had the average conductivity equal or greater than 0.4 to $1.5 \mu \mathrm{s} / \mathrm{cm}$ which indicate oil in water $(\mathrm{o} / \mathrm{w})$. Blank microemulsion and plant extract has 4.3 to $3.4 \mu \mathrm{s} / \mathrm{cm}$. The blank microemulsion and microemulsion containing extract had appropriate observed $\mathrm{pH}$ value (5.93 to 5.90) that is best for topical application. Incorporation of extract did not significantly affect the observed $\mathrm{pH}$ value of the microemulsions (Table-1). Skin $\mathrm{pH}$ values are variable as reported in literature ${ }^{13}$ with a broad range of $\mathrm{pH} 4$ to $7 . \mathrm{pH}$ of our formulations are well within the reported range of skin $\mathrm{pH}$.

The mean viscosity of formulations was from $5.54 \pm 0.12$ cps to $4.44 \pm 0.05 \mathrm{cps}$. There was no significant difference found between the viscosities of blank microemulsion and microemulsion extract containing $(\mathrm{p}>0.05)^{14}$. designed and characterized microemulsion systems for Naproxen and found viscosity in the range of $253.73 \mathrm{cPs}$ to $802.63 \mathrm{cP}$ which is much higher than our microemulsion system. This is because the proportion of surfactant to co-surfactant in $S_{\text {mix }}$ is $4: 1$ and $6: 1$ while in our microemulsion this is $2: 1$. Higher the amount of $S_{\text {mix }}$, higher will be the viscosity.

The visual inspection and physical stability experiment was carried out for 3 months by drawing ME sample at weekly interval for the first month and monthly interval for the subsequent months. The visual observation showed no evidence of phase separation or any precipitation or flocculation. These samples also revealed no sign of phase separation under stress when subjected to centrifugation at $8000 \mathrm{rpm}$ for $0.5 \mathrm{~h}$. The centrifugtion tests showed that microemulsions were remained homogenous without any phase separation throughout the test indicates good physical stability of both preparations ${ }^{15}$.

The spreadability is an important criterion for uniform and ease of application of transdermal preparations. Spreadability of the microemulsions was measured in terms of average diameter of the spread circle. As shown in Table-1, spreadability values for prepared microemulsions ${ }^{16,17}$ ranged between 5.62 and $5.81 \mathrm{~cm}$.

\section{Conclusion}

This study established that physico-chemical properties were dependent upon the contents of S/C ratio, oil and water in formulations. Stable microemulsion is prepared by incorporation of plant extract by studying physicochemical properties. Thus it is concluded that microemulsion containing extract is stable with 2:1 S/C, IPM and water. Further this formulation is used for cosmetically, topically for transdermal delivery of antioxidant, flavonols and polyphenolic components of herbal natural sources.

\section{ACKNOWLEDGEMENTS}

This paper is extracted from Ph.D. research work and financial support was provided by Higher Education Commission of Pakistan. The authors are very thankful to Chairman, Department of Pharmacy and Dean, Faculty of pharmacy and alternative medicine, Khawaja Farid Campus, the Islamia University of Bahawalpur for providing research facilities.

\section{REFERENCES}

1. E. Moghimipour, A. Salimi and S. Eftekhari, Adv. Pharm. Bull., 3, 63 (2013).

2. R. Rowe, P. Sheskey and M. Quinn, Handbook of Pharmaceutical Excipients, Pharmaceutical Press, Philadelphia, edn 6 (2009).

3. J.T. Davies, Proceedings of $2^{\text {nd }}$ International Congress Surface Activity, Butterworths, London, vol. 1, pp. 426-438 (1959).

4. S.K. Hait and S.P. Moulik, J. Surfact. Deterg., 4, 303 (2001).

5. C.D. Luke, F. Kent, G.D. Ralf and A.H. Timothy, Journal of Experimental Botany, (2009).

6. U.G. Chandrika, E.R. Jansz, S.M.D.N. Wrikamasinghe and N.D. Warnasuriya, J. Natn. Sci Found. Sri Lanka, 31, 437 (2003).

7. L.B. Lopes and R. Reed, Biomed. Chromatogr., 24, 154 (2009).

8. I.R. Bunghez, M. Raduly, S. Doncea, I. Aksahin and R.M. Ion, Digest J. Nanomater. Biostruct., 6, 1349 (2011).

9. K.G.H. Desai, Drug Dev. Res., 63, 33 (2004).

10. S.K. Mehta, G. Kaur and K.K. Bhasin, AAPS Pharm. Sci. Tech., 11, 143 (2010).

11. N. Kashyap, P. Jadon, P. Naruka and C. Chauhan, J. Chem. Pharm. Res., 2, 357 (2010).

12. P. Katakam and C. Narendra, Asian J. Pharm. Life Sci., 1, 354 (2011).

13. H. Lambers, S. Piessens, A. Bloem, H. Pronk and P. Finkel, Int. J. Cosmet. Sci., 28, 359 (2006).

14. E. Moghimipour, A. Salimi and F. Leis, Adv. Pharm. Bull., 2, 141 (2012).

15. K. Jadhav, S. Shetye and V. Kadam, Int. J. Adv. Pharm. Sci., 1, 156 (2011).

16. A. Garg, D. Aggarwal, S. Garg and A. K. Singla, Pharm. Technol., 26, 84 (2002).

17. N. Waghmare, P. Waghmare, S. Wani and A. Yerawar, Res. J. Pharm. Biol. Chem. Sci., 2, 220 (2011). 